\title{
Einleitung.
}

\section{Geschichte der Edition.}

Das Ms. der Pistis Sophia ist in der gelehrten Welt unter dem Namen Codex Askewianus bekannt. Es trägt diesen Namen nach dem ersten Besitzer Dr. Askew, aus dessen Nachlaß der Kodex im Jahre 1785 von dem British Museum für den geringen Preis von $\mathscr{E} 10 \cdot 10$ erworben wurde. Auf welchem Wege das Ms. in den Besitz von Dr. Askew gekommen ist, steht nicht fest. Nach der Mitteilung von Woide in einem Briefe an Michaelis vom Jahre $1773^{1}$ soll es bei einem Buchhändler - d. h. höchstwahrscheinlich in London gekauft sein. Daraus geht die eine Tatsache hervor, daß Dr. Askew es nicht in Ägypten selbst erworben hat. Die Notiz von Koestlin, Theol. Jahrbücher, herausgeg. von Baur und Zeller, 1854, S. 1, Anm. 1, über das Brittische theolog. Magazin vom Jahre 1770 , Bd. 1, Stück 4, S. 223, ist jetzt geklärt, nachdem ich ein Exemplar dieser seltenen Zeitschrift auf der Staatsbibliothek aufgespürt habe. ${ }^{2}$ Herr Woide,

1 Vgl. Buhle, Literarischer Briefwechsel von Joh. David Michaelis (Leipzig). Vol. III (1796), p. 69.

2 Die Abhandlung ist betitelt: „Nachricht von dem Büchervorrath des Herrn Dr. Anton Askew in London, und von einigen griechischen Handschriften, die sich darin befinden." Daselbst lesen wir, daß Dr. Askew ein Arzt in London, Mitglied der königl. Londonschen Sozietät der Wissenschaften and anderer gelehrten Gesellschaften war. Er soll über 300 Pergamenthandschriften in lateinischer und griechischer Sprache in seiner kostbaren Bibliothek vereinigt haben, die sich besonders anf die Kirchenväter, die Bibel and die Arzneiwissenschaft bezogen. Auch von einer riesigen Sammlung altester und erster Ausgaben lateinischer und griechischer Schriftsteller und Kirchenväter spricht der Berichterstatter. Die Hds. soll Dr. Askew auf seinen Reisen in Italien, Griechenland und besonders auf dem Berge Athos und anderen Orten erworben haben. Das ist die Notiz, anf die Koestlin anspielt, aber sie hat nichts zu tun mit unserem koptischen 
nach der Angabe von Legge damals Minister at the German Chapel at St. James Palace, bekannt als der Herausgeber des Cod. Alexandrinus, der sich in jenen Tagen mit koptischen Studien beschäftigte, hatte die erste Gelegenheit, bei dem ursprünglichen Besitzer das Ms. einsehen za können und lenkte die Aufmerksamkeit der Bibelforscher auf das Werk in einer Abhandlung, abgedruckt in J. A. Cramers Beyträge zur Beförderung theologischer und andrer wichtiger Kenntnisse (Kiel und Hamburg) 1778, III, S. $55 \mathrm{f}$. und $154 \mathrm{f}$. Er führte die Schrift unter dem Namen Pistis Sophia ein, der seitdem allgemein üblich geblieben ist. Ferner verwertete er die vorkommenden neutestamentlichen Zitate in seinem großen Werke: Appendix ad editionem Novi Testamenti Graeci e codice Ms. Alexandrino a Carolo Godofredo Woide descripti, in qua continentur fragmenta Novi Testamenti juxta interpretationem dialecti superioris Aegypti, quae Thebaidica vel Sahidica appellatur, e codicibus Oxoniensibus maxima ex parte desumpta, cum dissertatione de versione Bibliorum Aegyptiaca, quibus subjicitur codicis Vaticani collatio, Oxonii 1799.

Woide hatte eine Abschrift von dem Ms. genommen, denn er berichtet in Cramers Beiträgen p. 84: "Die sehr alte Handschrift hat mir Herr Dr. Askew and seine Erben ${ }^{1}$ so lange gelehnet, daß ich sie völlig habe abschreiben und meine Abschrift mit dem Original vergleichen können", aber zu einer Publikation ist es nicht gekommen. Angeregt durch dessen Dissertatio, p. 148 sq., veröffentlichte im Jahre 1812 der dänische Bischof Münter, sicherlich nach einer $\mathrm{Ab}$ schrift von Woide, die in der Pistis Sophia erhaltenen fünf pseudo-salomonischen Oden: Odae gnosticae Salomoni tributae thebaice et latine, praefatione et adnotationibus philologicis illustratae. Havniae 1812. Diese Oden sind, um dies hier schon vorwegzunehmen, durch die Entdeckung einer syrischen

Kodex. Am Schluß folgt nämlich ein Verzeichnis der griechischen Hds. des Neuen Testaments nebst einem lateinischen Kodex der vier Evangelien. - Diese gelehrte Notiz stammt ohne Zweifel aus der Feder von Woide, der ja gerade für neutestamentliche Handschriften ein großes Interesse hatte.

1 Nach den liebenswürdigen Bemühungen von Herrn Mead ist Dr. Askew im J. 1772 zu Hampstead in der Nähe von London verstorben. Die erste Auktion seiner Bibliothek im J. 1775 brachte die Summe von $\mathscr{E} 3993$ und die zweite im J. 1785 die Summe von $£ 1277$. 
Handschrift von Rendel Harris ${ }^{1}$ als ein Teil der 42 alten Oden Salomos festgestellt worden; dadurch erledigt sich die frühere Literatur darüber. ${ }^{2}$ Während seines Aufenthaltes in England in den Jahren 1838-1840 konnte D u la urier eine Abschrift des Ms. nehmen; er stellte auch eine Publikation mit vollständigem Glossar in Aussicht, ${ }^{3}$ aber der Druck ist nicht ausgeführt. Dulauriers Ms. wird jetzt in seinem literarischen Nachla $B$ auf der Bibliothèque Nationale aufbewahrt.

Da wurde im Jahre 1848 der junge Prof. M. G. Sch wartze im Auftrage der Königl. Preuß. Akademie der Wissenschaften zum Studium koptischer Handschriften nach England geschickt. Es war nur zu natürlich, daß er sich auf den Cod. Askewianus stürzte und eine Abschrift anfertigte. Nach seiner Rückkehr begann er sofort mit den Vorbereitungen der Edition, aber an der Publikation selbst ist er durch den frühzeitigen Tod verhindert worden. Diese war glücklicherweise so weit druckfertig, daß sein intimer Freund und Kollege Prof. J. H. Peterm a n n, der bekannte Orientalist, die Ausgabe besorgen konnte, die dann im Jahre 1851 unter dem Titel erschien: Pistis Sophia, opus gnosticum Valentino adiudicatum e codice manuscripto coptico Londinensi descripsit et latine vertit M. G. Schwartze, edidit J. H. Petermann, Berolini, 1851. Petermann hat sich darauf beschränkt, das zarückgelassene Ms. sorgfältig durchzuarbeiten und offensichtliche Fehler auszumerzen, auch hat er für den Text einige Verbesserungen beigesteuert und besonders die Übersetzung revidiert. Auf diese Weise kann der Editio princeps trotz mancherlei Mängel - besonders störend ist die eigentümliche Trennung der Wörter - nur uneingeschränkte Anerkennung gespendet werden. Die Akribie der Abschrift und die Sorgfalt der Übersetzung bedeuten in Rücksicht auf den damaligen Stand der koptischen Wissen-

1 The Odes and Psalms of Solomon, now first published from the Syriac Version. Cambridge 1909. Dazu Worrell, The Odes of Solomon and the Pistis Sophia in Journ. of Theolog. Stud. XIII (1912), S. $29 \mathrm{ff}$.

2 R y le and J a mes, The Psalms of Solomon, Cambridge 1891, S. $155 \mathrm{ff}$; H a r n a c k, Über das guostische Buch Pistis Sophia (T. u. U., Bd. VII, 2, S. 35 ff.).

3 Notice sur le manuscrit copte-thébain intitulé: La Fidèle Sagesse (tpistis sophia) et sur la publication projetée da texte et de la traduction française de ce manuscript in dem Journal Asiatiqne, quatrième série, tom. IX, 1847, p. $534 \mathrm{ff}$. 
schaft eine hervorragende Leistung; ja man könnte sogar der Meinung sein, eine erneute Publikation wäre überflüssig, wenn nicht der größte Teil der unverkauften Exemplare eingestampft wäre, so daß in antiquarischen Buchhandel nur selten ein Exemplar aufzutreiben ist. Schwartze hatte seine Übersetzung in lateinischer Form mit Beibehaltung der überaus zahlreichen griechischen Wörter veröffentlicht. Eine Übersetzung in moderner Sprache erschien zuerst aus der Feder

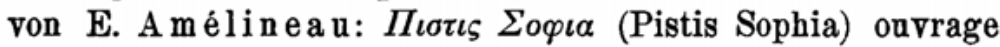
gnostique de Valentin, traduit du Copte en Français avec une introduction [Les classiques de l'Occulte], Paris $1895 .^{1}$ Auf der Grundlage der Amélineauschen Übersetzung veröffentlichte G. R. S. Me a d, der jetzige Vorsitzende der englischen Theosophischen Gesellschaft und Herausgeber der Zeitschrift „The Quest", sein Buch: Pistis Sophia: A gnostic gospel (with extracts from the books of the Saviour appended), originally translated from Greek into Coptic and now for the first time Englished from Schwartzes Latin version on the only known Coptic MS. and checked by Amélineau's French version, London, $1896 .^{2}$ Sehr nützlich ist die Einleitung wegen ihrer vortrefflichen Orientierung über die erschienene Literatur und die verschiedenen Meinungen der Gelehrten. Inzwischen war ich selbst von der KirchenväterKommission der Akademie der Wissenschaften mit der Übersetzung der koptisch-gnostischen Schriften betraut worden, und es erschien die Pistis Sophia zusammen mit den in dem Papyrus Brucianus enthaltenen gnostischen Schriften in den „Koptisch-Gnostischen Schriften“, Bd. I, Leipzig 1905. Diese Übersetzung gab Herrn M e ad die Veranlassung, sein früheres Werk in neuem Gewande erscheinen zu lassen unter dem Titel: Pistis Sophia. A gnostic miscellany: being for the most part extracts from the books of the Saviour, to which are added excerpts from a cognate literature. Englished

1 Diese Übersetzang, insbesondere die einleitenden Untersuchungen genügen nicht allen berechtigten Anforderungen. Vgl. meine Anzeige in den Gött. Gel. Anz., 1898, Nr. 6, S. 436 ff. und An ders son in der "Sphinx “, Bd. VIII, S. 237 ff.; IX, S. 52 ff.; S. $233 \mathrm{ff} . ;$ X, S. 44 ff.; XI, S. $156 \mathrm{ff}$.

2 Vorher soll C. W. King, der Herausgeber des Werkes The Gnostics and their Remains (2. Ed. 1887), eine englische Übersetzung geplant haben, die aber nicht druckfertig im Nachlaß vorgefunden wurde. 
(with an introduction and annotated bibliography). London 1921. Eine neue englische Übersetzung hat soeben die Presse verlassen. Sie ist unter dem Titel erschienen: Pistis Sophia. Literally translated from the Coptic by George Horner. With an introduction by F. Legge, F. S. A., London 1924, Society for promoting christian knowledge. ${ }^{1}$ So werden die interessierten Kreise, welche ihre Kenntnis der gnostischen Ideenwelt nicht aus dem Original selbst schöpfen können, an der Hand der Übersetzungen in den Stoff einzudringen Gelegenheit finden. Freilich werden sie zugleich vor der Tatsache stehen, dab Legges und meine Ansichten über Alter, Verfasser, Komposition usw. sich ganz diametral gegenüberstehen, sicherlich ein beklagenswerter Zustand in den Augen des gebildeten Laienpublikums.

\section{Beschreibung der Handschrift. ${ }^{2}$}

Der Codex Askewianus wird heute in einem modernen Einbande unter M. S. Add. 5114 im British Museum aufbewahrt. Auf dem eingesetzten Vorsatzblatte lesen wir die handschriftliche Eintragung, die nach Meads Vermutung von Woide herrührt: Codex dialecti Superioris Aegyti, quam Sahidicam seu Thebaidicam vocant, cuius titulus exstat pagina 115: Pmeh snaou ìtomos ǹtpiste Sophia - Tomos secundus fidelis Sapientiae - deest pagina 337-344. Das Ms. ist auf feinem Pergament geschrieben und umfaßte ur-

1 Der Vorrede zafolge hatte L e g ge, der in seinem Werke Forerunners and Rivals of Christianity : Being Studies in religious history from 330 B. C. to 330 A. D. (Cambridge) 2 vols., 1915, sich mit der Pistis Sophia beschäftigt hatte, die von Horner besorgte wörtliche Übersetzung mit ausführlichen Sacherklärungen versehen. Diese sind nach dessen Tode nicht zum Abdruck gebracht, sondern als selbständige Abhandlung ist nur die Introduction verblieben, für die also $\mathrm{Leg} g \mathrm{e}$ allein die Verantwortung trägt. M. E. hätte es nichts geschadet, wenn auch diese den Lesern vorenthalten wäre, da sie den Wert der Publikation stark herabdrückt.

2 Vergleiche die kurze Beschreibung von Woide in der Dissertatio de versione Bibliorum aegyptiaca, p. 19 seines oben genannten Appendix, und vorher in seinen Beiträgen, S. 83. Ferner Crum, Egyptian Exploration Fund, Archaeological Report, 1897/98, p. 62 und Catalogue of the Coptic MSS. in the British Museum, London 1905, p. 173 unter Nr. 367; anch Coptic Mss. brought from the Fayûm, London 1893, p. 3, Note. - Bei der Beschreibung der Hds. muß ich auf die Textausgabe Rücksicht nehmen. 